Бегун А. С., Ковтанюк Л. В., Лемза А. О.

A. S. Begun, L. V. Kovtanyuk, A. O. Lemza

МОДЕЛИРОВАНИЕ ПРОЦЕССА ПРОИЗВОДСТВА БОЛЬШИХ

ВИСКОЗИМЕТРИЧЕСКИХ ДЕФОРМАЦИЙ ВЯЗКОУПРУГОГО МАТЕРИАЛА ПРИ ЕГО ОДНОСТОРОННЕМ ПРОСКАЛЬЗЫВАНИИ

\title{
MODELING OF THE PRODUCTION PROCESS OF LARGE VISCOZYMETRIC DEFORMATION OF VISCOELASTIC MATERIAL WHEN IT IS ONE SIDE SLIPPING
}

Бегун Александра Сергеевна - кандидат физико-математических наук, научный сотрудник лаборатории механики необратимого деформирования Института автоматики и процессов управления Дальневосточного отделения Российской академии наук (Россия, Владивосток); 690041, Приморский край, г. Владивосток, ул. Радио, 5. E-mail: asustinova@mail.ru.

Ms. Alexandra S. Begun- PhD in Phys. \& Math., Researcher of the Laboratory of Irreversible Deformation Mechanics, Institute of Automation and Control Processes, Far East Branch of the Russian Academy of Sciences (Russia, Vladivostok); 690041, Primorsky territory, Vladivostok, 5 Radio str. E-mail: asustinova@mail.ru.

Ковтанюк Лариса Валентиновна - доктор физико-математических наук, заведующая лабораторией механики необратимого деформирования Института автоматики и процессов управления Дальневосточного отделения Российской академии наук (Россия, Владивосток); 690041, Приморский край, г. Владивосток, ул. Радио, 5. E-mail: 1k@iacp.dvo.ru.

Ms. Larisa V. Kovtanyuk - Dr. Sci. Phys. \& Math., Head of the Laboratory of Irreversible Deformation Mechanics, Institute of Automation and Control Processes, Far East Branch of the Russian Academy of Sciences (Russia, Vladivostok); 690041, Primorsky territory, Vladivostok, 5 Radio str. E-mail: 1k@iacp.dvo.ru.

Лемза Александр Олегович - аспирант Дальневосточного федерального университета (Россия, Владивосток). E-mail: alsu-24@yandex.ru.

Mr. Alexander O. Lemza - Postgraduate Student, Far Eastern Federal University (Russia, Vladivostok). E-mail: alsu-24@yandex.ru.

Аннотация. В статье в рамках теории больших деформаций рассматривается деформирование материала с нелинейными упругими и вязкими свойствами, находящегося в зазоре между двумя жёсткими коаксиальными цилиндрическими поверхностями при вращении одной из жёстких поверхностей и при неподвижности другой. Исследован случай проскальзывания материала в окрестности внутренней стенки. Рассматривается равноускоренное движение вращающегося цилиндра, последующие его движения с постоянной скоростью и дальнейшее равнозамедленное движение до остановки. Рассчитаны напряжения, обратимые и необратимые деформации, перемещения; исследована релаксация напряжений после полной остановки цилиндра. Решение задачи получено в программном комплексе, разработанном на базе системы компьютерной математики Scilab.

Summary. This paper considers a deformation process of a material with nonlinear elastic and viscous properties in the framework of the theory of large strains. The material is placed between two rigid coaxial cylindrical surfaces and deformed at the rotation of one of them while the other one is motionless. The case of material slipping inside the internal surface is investigated. The motion of a rotating cylinder is considered uniformly accelerated, at constant velocity and uniformly decreasing until rotation stops sequentially. Stresses, reversible and irreversible strains, displacements are calculated; the stress relaxation after the stop of cylinder rotation is evaluated. The present problem is solved in the software complex developed on the base of the system of computer mathematics Scilab.

Ключевые слова: большие деформации, упругость, ползучесть, проскальзывание, релаксация напряжений. Key words: large strains, elasticity, creep, slipping, stress relaxation.

УДК 004.942 
Бегун А. С., Ковтанюк Л. В., Лемза А. О.

МОДЕЛИРОВАНИЕ ПРОЦЕССА ПРОИЗВОДСТВА БОЛЬШИХ ВИСКОЗИМЕТРИЧЕСКИХ ДЕФОРМАЦИЙ ВЯЗКОУПРУГОГО МАТЕРИАЛА

ПРИ ЕГО ОДНОСТОРОННЕМ ПРОСКАЛЬЗЫВАНИИ

\section{Введение}

Изменение формы некоторых конструкционных материалов осуществляется способом холодной формовки в условиях ползучести [7]. Этим вызван интерес к постановкам задач теории больших деформаций, когда при кинематическом воздействии на деформируемые материалы необратимые деформации накапливаются в условиях ползучести при низкой температуре. Здесь рассмотрим такой случай на примере задачи о вискозиметрическом деформировании несжимаемого материала, образующего слой между двумя жёсткими коаксиальными цилиндрическими поверхностями, при условии возможного проскальзывания в окрестности внутренней жёсткой стенки.

\section{Основные модельные соотношения}

В настоящей работе для моделирования поведения среды используется теория больших упругопластических деформаций, в которой разделение деформаций на обратимую и необратимую составляющие определяется дифференциальными уравнениями изменения (переноса) [4; 5].

В прямоугольной системе пространственных декартовых координат Эйлера $x_{i}$ кинематика среды определяется соотношениями

$$
\begin{gathered}
d_{i j}=\frac{1}{2}\left(u_{i, j}+u_{j, i}-u_{k, i} u_{k, j}\right)=e_{i j}+p_{i j}-\frac{1}{2} e_{i k} e_{k j}-e_{i k} p_{j k}-p_{i k} e_{j k}+e_{i k} p_{m k} e_{j m}, \\
\frac{D e_{i j}}{D t}=\varepsilon_{i j}-\gamma_{i j}-\frac{1}{2}\left[\left(\varepsilon_{i k}-\gamma_{i k}+z_{i k}\right) e_{k j}+e_{i k}\left(\varepsilon_{k j}-\gamma_{k j}-z_{k j}\right)\right], \\
\frac{D p_{i j}}{D t}=\gamma_{i j}-p_{i k} \gamma_{k j}-\gamma_{i k} p_{k j}, \quad \frac{D n_{i j}}{D t}=\frac{d n_{i j}}{d t}-r_{i k} n_{k j}+n_{i k} r_{k j}, \\
\varepsilon_{i j}=\frac{1}{2}\left(\mathrm{v}_{i, j}+\mathrm{v}_{j, i}\right), \quad \omega_{i j}=\frac{1}{2}\left(\mathrm{v}_{i, j}-\mathrm{v}_{j, i}\right), \quad \mathrm{v}_{i}=\frac{d u_{i}}{d t}=\frac{\partial u_{i}}{\partial t}+u_{i, j} \mathrm{v}_{j}, \quad u_{i, j}=\frac{\partial \mathrm{u}_{\mathrm{i}}}{\partial x_{j}},
\end{gathered}
$$

где $d_{i j}$ - компоненты тензора деформаций Альманси; $u_{i}$ - компоненты вектора перемещений точек среды: $e_{i j}$ и $p_{i j}$ - обратимая (упругая) и необратимая составляющие $d_{i j}$ соответственно; $\frac{D n_{i j}}{D t}$ оператор используемой объективной производной произвольного тензора $n_{i j}$ по времени; $\varepsilon_{i j}$ и $\gamma_{i j}-$ компоненты тензоров скоростей полных и необратимых деформаций соответственно; $\omega_{i j}-$ кососимметричный тензор вихря скорости; $v_{i}$ - компоненты вектора скорости точек среды, а $r_{i j}-$ тензор вращений, который вместе со своей нелинейной составляющей $z_{i j}$ определяется следующим образом:

$$
\begin{gathered}
r_{i j}=\omega_{i j}+z_{i j}, z_{i j}=A^{-1}\left[\left(\varepsilon_{i m} e_{m j}-e_{i m} \varepsilon_{m j}\right) B^{2}+B\left(\varepsilon_{i m} e_{m n} e_{n j}-\mathrm{e}_{i m} e_{m n} \varepsilon_{n j}\right)+e_{i m} \varepsilon_{m n} e_{n k} e_{k j}-e_{i m} e_{m n} \varepsilon_{n k} e_{k j}\right], \\
A=8-8 E_{1}+3 E_{1}^{2}-E_{2}-\frac{1}{3} E_{1}^{3}+\frac{1}{3} E_{3}, B=2-E_{1}, E_{1}=e_{k k}, E_{2}=e_{i j} e_{j i}, E_{3}=e_{i j} e_{j k} e_{k i} .
\end{gathered}
$$

Когда $\gamma_{i j}=0$, компоненты $p_{i j}$ изменяются так же, как при повороте системы координат, или как при движении среды без деформирования, то есть $\frac{D p_{i j}}{D t}=0$. При $z_{i j}=0$ производная в (1) переходит в производную Яумана.

Полагаем, что термодинамический потенциал (плотность распределения свободной энергии) является изотропной функцией только обратимых деформаций. В таком случае, следуя закону сохранения энергии, напряжения в среде однозначно определяются обратимыми деформациями и связаны с ними зависимостью, аналогичной формуле Мурнагана, известной в нелинейной теории упругости [6]. Запишем такую зависимость для несжимаемой среды: 


\begin{tabular}{|c|c|}
\hline комсомольского-на-Амуре государственного техническогоуниверситета \\
\hline$\sigma_{i j}=-p \delta_{i j}+\frac{\partial W}{\partial e_{i k}}\left(\delta_{k j}-e_{k j}\right)$
\end{tabular}

где $\sigma_{i j}$ - компоненты тензора напряжений Эйлера - Коши; $p$ - добавочное гидростатическое давление; $W$ - упругий потенциал, задаваемый для изотропной среды в следующем виде:

$$
\begin{gathered}
W=-2 \mu I_{1}-\mu I_{2}+b I_{1}^{2}+(b-\mu) I_{1} I_{2}-\chi I_{1}^{3}+\ldots, \\
I_{1}=e_{k k}-\frac{1}{2} e_{i j} e_{j i}, \quad I_{2}=e_{i k} e_{k i}-e_{i j} e_{j k} e_{k i}+\frac{1}{4} e_{i j} e_{j k} e_{k l} e_{l i},
\end{gathered}
$$

где $\mu$ - модуль сдвига, присущий материалу; $b, \chi$ - упругие константы высшего порядка. Диссипативный механизм деформирования, определяющий накопление необратимых деформаций, связан с реологическими и пластическими свойствами материала.

Далее полагаем, что необратимые деформации связаны со свойством ползучести материала и накапливаются с начала деформационного процесса, а пластическое течение в ходе деформирования не наблюдается. Чтобы задать соответствующий диссипативный механизм, выберем термодинамический потенциал $V\left(\sigma_{i j}\right)$ в форме степенного закона ползучести Нортона [8]:

$$
V\left(\sigma_{i j}\right)=B \Sigma^{n}\left(\sigma_{1}, \sigma_{2}, \sigma_{3}\right), \Sigma=\max \left|\sigma_{i}-\sigma_{j}\right|, \quad \gamma_{i j}=\varepsilon_{i j}^{v}=\frac{\partial V}{\partial \sigma_{i j}},
$$

где $B, n$ - параметры ползучести материала; $\sigma_{k}$ - главные значения тензора напряжений; $\varepsilon_{i j}^{v}-$ тензор скоростей деформаций ползучести. Учитывая, что возникновение и развитие пластического течения в материале не рассматривается, напряжённое состояние в таком случае не должно достигать поверхности нагружения. Принимая в качестве таковой условие пластичности максимального касательного напряжения, получим, что в течение рассматриваемого процесса деформирования должно быть справедливо неравенство $\max \left|\sigma_{i}-\sigma_{j}\right|<2 k$, где $k-$ предел текучести материала.

\section{Постановка задачи, поворот внешнего цилиндра}

Рассмотрим деформирование несжимаемого материала, составляющего слой между двумя жёсткими коаксиальными цилиндрическими поверхностями при повороте одной из них с переменной скоростью, тогда как другая остаётся неподвижной.

Пусть для определённости вращается внешний цилиндр радиуса $r=R$, а внутренняя поверхность радиуса $r=r_{0}$ неподвижна.

Для решения задачи будем использовать цилиндрическую систему координат $(r, \varphi, z)$. Считая, что все точки среды движутся по окружностям, определим кинематику среды согласно (1) следующими соотношениями:

$$
\begin{gathered}
u_{r}=r(1-\cos \theta), \quad u_{\varphi}=r \sin \theta, \quad d_{r r}=-\frac{1}{2}\left(r \frac{\partial \theta}{\partial r}\right)^{2}, \quad d_{r \varphi}=\frac{1}{2} r \frac{\partial \theta}{\partial r}, \\
\mathrm{v}_{\varphi}=r \frac{\partial \theta}{\partial t}=r w, \quad \varepsilon_{r \varphi}=\frac{1}{2} r \frac{\partial^{2} \theta}{\partial r \partial t}=\varepsilon_{r \varphi}^{e}+\varepsilon_{r \varphi}^{v}, \quad \omega_{r \varphi}=-\varepsilon_{r \varphi}-\frac{\partial \theta}{\partial t}, \quad r_{\varphi r}=\frac{\partial \theta}{\partial t}+\frac{2 \varepsilon_{r \varphi}\left(e_{\varphi \varphi}-1\right)}{e_{r r}+e_{\varphi \varphi}-2},
\end{gathered}
$$

где $\theta=\theta(r, t)$ - центральный угол закручивания; $\varepsilon_{r \varphi}^{e}-$ компоненты тензора скоростей обратимых деформаций. Скорость вращения внешнего цилиндра $w_{R}(t)$ зададим последовательно возрастающей, постоянной, убывающей и нулевой: 


$$
w_{R}(t)=\left\{\begin{array}{lc}
\alpha^{2} t, & 0 \leq t \leq t_{1}, \\
\alpha^{2} t_{1}, & t_{1} \leq t \leq t_{2}, \\
\alpha^{2} t_{1}-\beta^{2}\left(t-t_{2}\right), & t_{2} \leq t \leq t_{3}, \\
0, & t_{3} \leq t,
\end{array}\right.
$$

где $\alpha, \beta$ - задаваемые константы; $t_{k}$ - некоторые моменты времени.

На цилиндрических стенках выполняются условия прилипания, а напряжённое состояние вызывается начальным поджатием, поэтому граничные условия запишем в виде

$$
\left.\bar{u}\right|_{r=r_{0}}=\left.\overline{\mathrm{v}}\right|_{r=r_{0}}=0,\left.\quad \theta\right|_{r=R}=\theta_{R}(t)=\int_{0}^{t} w_{R}(\xi) d \xi,\left.\quad \sigma_{r r}\right|_{r=r_{0}}=\sigma_{0} .
$$

В качестве начальных условий выступает отсутствие деформаций и напряжений в начале деформирования.

Контакт материала с жёсткими стенками при $r=r_{0}$ и $r=R$ первоначально осуществляется согласно закону сухого трения [2]:

$$
\left|\sigma_{r \varphi}\right| \leq \gamma\left|\sigma_{r r}\right|
$$

где $\gamma$ - коэффициент трения покоя. Диагональные компоненты тензоров деформаций в задачах данного класса $[2 ; 3]$ являются малыми более высокого порядка по сравнению с недиагональными. Поэтому далее будем пренебрегать слагаемыми выше первого порядка по диагональным компонентам тензоров деформаций и выше второго - по недиагональным, что позволяет значительно облегчить последующие аналитический и вычислительный процессы. Тогда согласно (3) и (4) компоненты тензора напряжений можно определить в виде

$$
\begin{gathered}
\sigma_{r r}=-p-2 \mu+2(b+\mu) e_{r r}+2 b e_{\varphi \varphi}+\mu e_{r \varphi}^{2}, \\
\sigma_{\varphi \varphi}=-p-2 \mu+2(b+\mu) e_{\varphi \varphi}+2 b e_{r r}+\mu e_{r \varphi}^{2}, \\
\sigma_{z z}=-p-2 \mu+2 b\left(e_{r r}+e_{\varphi \varphi}\right)-2 \mu e_{r \varphi}^{2}, \\
\sigma_{r \varphi}=2 \mu e_{r \varphi},
\end{gathered}
$$

откуда

$$
\frac{\sigma_{r r}-\sigma_{\varphi \varphi}}{\sigma_{r \varphi}}=\frac{e_{r r}-e_{\varphi \varphi}}{e_{r \varphi}}
$$

Пренебрегая силами инерции, уравнения равновесия можно записать в форме

$$
\frac{\partial \sigma_{r r}}{\partial r}=\frac{\sigma_{\varphi \varphi}-\sigma_{r r}}{r}, \quad \frac{\partial \sigma_{r \varphi}}{\partial r}=-2 \frac{\sigma_{r \varphi}}{r} .
$$

Интегрирование последнего соотношения с учётом (10) приводит к следующим результатам:

$$
\sigma_{r \varphi}=\frac{c(t)}{r^{2}}, \quad e_{r \varphi}=\frac{c(t)}{2 \mu r^{2}} .
$$

Потенциал $V\left(\sigma_{i j}\right)$ в принятых выше ограничениях по компонентам тензоров деформаций имеет вид $V=B\left[\left(\sigma_{r r}-\sigma_{\varphi \varphi}\right)^{2}+4 \sigma_{r \varphi} \sigma_{\varphi r}\right]^{\frac{n}{2}}$. 
Тогда согласно (1), (5), (11) и (13) ненулевые компоненты тензора скоростей деформаций ползучести определяются соотношениями

$$
\varepsilon_{r \varphi}^{v}=\frac{\partial p_{r \varphi}}{\partial t}=B n 2^{n-1} \frac{c^{n-1}(t)}{r^{2 n-2}}, \quad \varepsilon_{r r}^{v}=\frac{e_{r r}-e_{\varphi \varphi}}{e_{r \varphi}} \frac{\varepsilon_{r \varphi}^{v}}{2}=-\varepsilon_{\varphi \varphi}^{v} .
$$

Осуществляя подстановку (13) и (14) в уравнение связи тензоров скоростей деформаций (6) и интегрируя получившийся результат при граничных условиях (8), поставленных на поверхности $r=r_{0}$, выведем дифференциальные уравнения для угла поворота:

$$
\theta(r, t)=\frac{c(t)}{2 \mu}\left(\frac{1}{r_{0}^{2}}-\frac{1}{r^{2}}\right)+\frac{B n 2^{n-1}}{n-1} c_{1}(t)\left(\frac{1}{r_{0}^{2 n-2}}-\frac{1}{r^{2 n-2}}\right)
$$

где

$$
c_{1}(t)=\int_{0}^{t} c^{n-1}(\xi) d \xi
$$

Используя граничные условия (8) на внешней цилиндрической поверхности, из (15) и (16) нетрудно получить уравнения для неизвестных функций $c(t)$ и $c_{1}(t)$ :

$$
\begin{aligned}
& \dot{c}(t)=\frac{2 \mu w_{R}(t)}{r_{0}^{-2}-R^{-2}}-\frac{\mu B n 2^{n}}{n-1} c^{n-1}(t) \cdot \frac{r_{0}^{2-2 n}-R^{2-2 n}}{r_{0}^{-2}-R^{-2}}, \quad c(0)=0, \\
& \dot{c}_{1}^{\frac{1}{n-1}}(t)=\frac{2 \mu \theta_{R}(t)}{r_{0}^{-2}-R^{-2}}-\frac{\mu B n 2^{n}}{n-1} c_{1}(t) \cdot \frac{r_{0}^{2-2 n}-R^{2-2 n}}{r_{0}^{-2}-R^{-2}}, \quad c_{1}(0)=0 .
\end{aligned}
$$

Известные численные распределения данных функций позволяют определить компоненты тензоров деформаций $e_{r \varphi}$ и $p_{r \varphi}$ с помощью (13) и (14) соответственно. Оставшиеся компоненты могут быть рассчитаны с использованием зависимостей, основанных на (1):

$$
\begin{gathered}
\frac{\partial p_{\varphi \varphi}(r, t)}{\partial t}=\varepsilon_{r \varphi}^{v} \frac{e_{r \varphi}^{2}-p_{\varphi \varphi}}{e_{r \varphi}}+\frac{4 p_{r \varphi} \varepsilon_{r \varphi}}{e_{r \varphi}^{2}+2}\left(1+p_{\varphi \varphi}-\frac{1}{2} e_{r \varphi}^{2}-2 e_{r \varphi} p_{r \varphi}\right), \\
p_{r r}=-p_{\varphi \varphi}-2 p_{r \varphi}^{2}, \quad e_{r r}=p_{\varphi \varphi}-\frac{3}{2} e_{r \varphi}^{2}-2 e_{r \varphi} p_{r \varphi}, \quad e_{\varphi \varphi}=-e_{r r}-e_{r \varphi}^{2} .
\end{gathered}
$$

Для отыскания диагональных компонент тензора напряжений требуется сначала определить $\sigma_{r r}$ путём решения первого уравнения равновесия (12) при условии (8), после чего осуществить последовательный поиск гидростатического давления $p$, а затем и самих напряжений посредством (10).

\section{Проскальзывание в окрестности внутреннего цилиндра}

Уравнения (15) и (17) справедливы при выполнении неравенства (9), однако с ростом напряжённого состояния в материале в некоторый момент времени $t_{s}$ в окрестности поверхности $r=r_{0}$ неравенство обратится в равенство. В таком случае при $t \geq t_{s}$ должно быть поставлено новое граничное условие при $r=r_{0}$ :

$$
\left|\sigma_{r \varphi}\left(r_{0}, t\right)\right|=\gamma\left|\sigma_{r r}\left(r_{0}, t\right)\right|+\xi w\left(r_{0}, t\right),
$$


Бегун А. С., Ковтанюк Л. В., Лемза А. О.

МОДЕЛИРОВАНИЕ ПРОЦЕССА ПРОИЗВОДСТВА БОЛЬШИХ ВИСКОЗИМЕТРИЧЕСКИХ ДЕФОРМАЦИЙ ВЯЗКОУПРУГОГО МАТЕРИАЛА

ПРИ ЕГО ОДНОСТОРОННЕМ ПРОСКАЛЬЗЫВАНИИ

где $\xi$ - постоянная вязкого трения; $w\left(r_{0}, t\right)$ - угловая скорость точек среды в окрестности $r=r_{0}$, а коэффициент $\gamma$ выбирается таким образом, что $\gamma\left|\sigma_{0}\right|<k$, так как пластическое течение отсутствует согласно постановке задачи. Полагаем при этом увеличение напряжений таким, чтобы проскальзывание не происходило в окрестности $r=R$.

Смена граничного условия приведёт к изменению дифференциального уравнения для функции $c(t)$.

Так, применяя к (6) граничное условие (8), заданное на $r=R$, получим формулу для вычисления угла поворота:

$$
\theta(r, t)=\theta_{R}(t)-\frac{c(t)}{2 \mu}\left(\frac{1}{r^{2}}-\frac{1}{R^{2}}\right)-\frac{B n 2^{n-1}}{n-1} c_{1}(t)\left(\frac{1}{r^{2 n-2}}-\frac{1}{R^{2 n-2}}\right)
$$

откуда при дифференцировании по $t$ получим уравнение для скоростей точек цилиндрического слоя:

$$
w(r, t)=w_{R}(t)-\frac{\dot{c}(t)}{2 \mu}\left(\frac{1}{r^{2}}-\frac{1}{R^{2}}\right)-\frac{B n 2^{n-1}}{n-1} c^{n-1}(t)\left(\frac{1}{r^{2 n-2}}-\frac{1}{R^{2 n-2}}\right) .
$$

Подставляя полученный результат при $r=r_{0}$ в уравнение (19), найдём новое дифференциальное уравнение для функции $c(t)$ :

$$
\dot{c}(t)=\frac{2 \mu}{r_{0}^{-2}-R^{-2}}\left[\frac{\gamma\left|\sigma_{0}\right|}{\xi}-\frac{c(t)}{\xi r_{0}^{2}}+w_{R}(t)\right]-\frac{\mu B n 2^{n}}{n-1} c^{n-1}(t) \frac{r_{0}^{2-2 n}-R^{2-2 n}}{r_{0}^{-2}-R^{-2}} .
$$

В качестве начального условия для (22) используется значение $c\left(t_{s}\right)$, вычисленное согласно (17). Функция $c_{1}(t)$ наиболее просто может быть численно определена здесь с помощью (16). Смена граничного условия не отражается на зависимостях, позволяющих определять компоненты тензоров деформаций (13), (14), (18) и напряжений (10), (12). Уравнения(19)-(22) действуют при $t_{s} \leq t \leq t_{a}$, где $t_{a}$ - некоторый момент времени, когда неравенство (9) станет снова справедливым. При $t \geq t_{a}$ для определения функций $c(t)$ и $c_{1}(t)$ станут действительны уравнения (17), подлежащие решению при начальных условиях $c\left(t_{a}\right)$ и $c_{1}\left(t_{a}\right)$, найденных из (16) и (22).

\section{Результаты решения задачи}

Решение представленной задачи осуществлено в программном комплексе, разработанном на базе свободно распространяемой системы компьютерной математики Scilab [1].

Расчёт проведён в безразмерных переменных $\tau=\alpha t$ и $\widetilde{r}=r / R$ при следующих заданных параметрах:

$$
B=3,5, n=3, \frac{k}{\mu} \approx 0,00245, \frac{b}{\mu}=4, \frac{\alpha}{\beta}=3, \frac{r_{0}}{R}=0,5, \frac{\xi \alpha}{\mu}=0,01, \frac{\gamma\left|\sigma_{0}\right|}{\mu} \approx 0,00046 .
$$

Графические результаты решения задачи представлены ниже.

На рис. 1 показано распределение угла поворота $\theta(r, t)$ в моменты времени $\tau_{1}, \tau_{2}, \tau_{3}$ и $\tau_{4}=30 \tau_{3}$ (спустя длительный промежуток времени после остановки вращения внешнего цилиндра). 


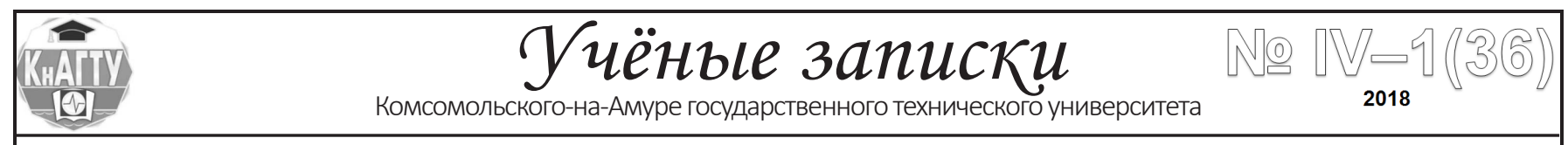

a)

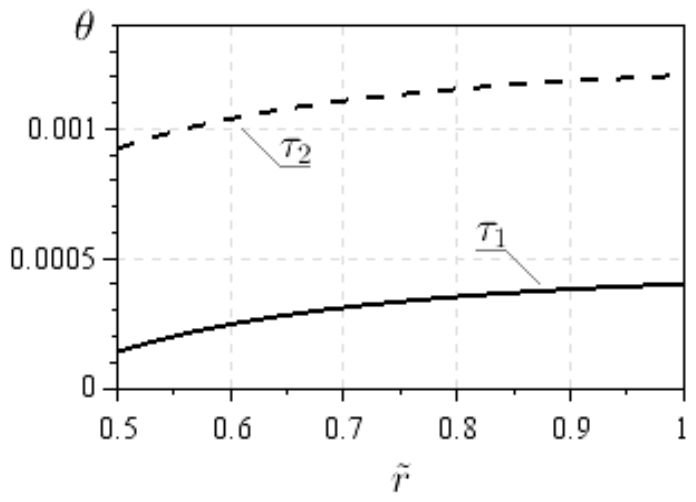

б)

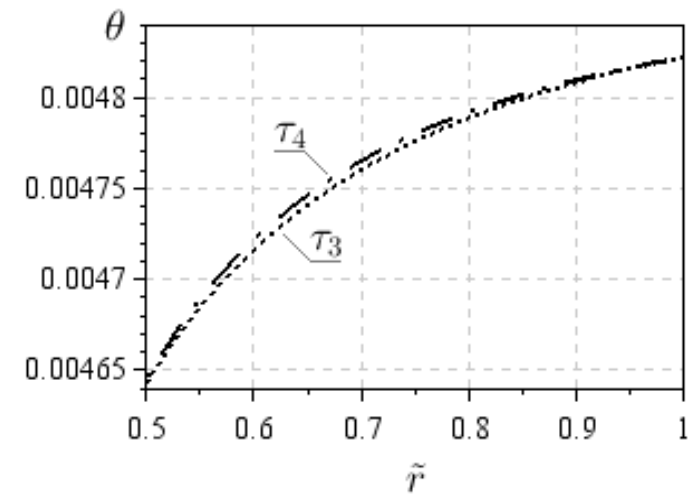

Рис. 1. Угол поворота $\theta(\widetilde{r}, \tau): \mathrm{a}-$ при $\tau=\tau_{1}$ и $\tau=\tau_{2} ; б-$ при $\tau=\tau_{3}$ и $\tau=\tau_{4}$

Изменения компонент тензора необратимых деформаций со временем у поверхности внутреннего цилиндра можно проследить на рис. 2.

a)

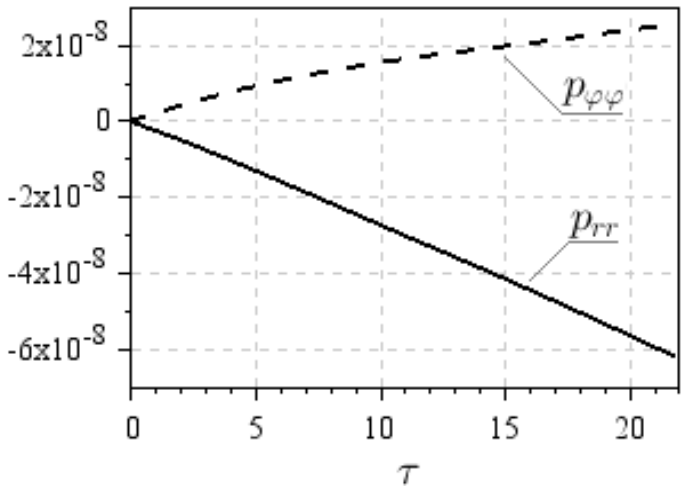

б)

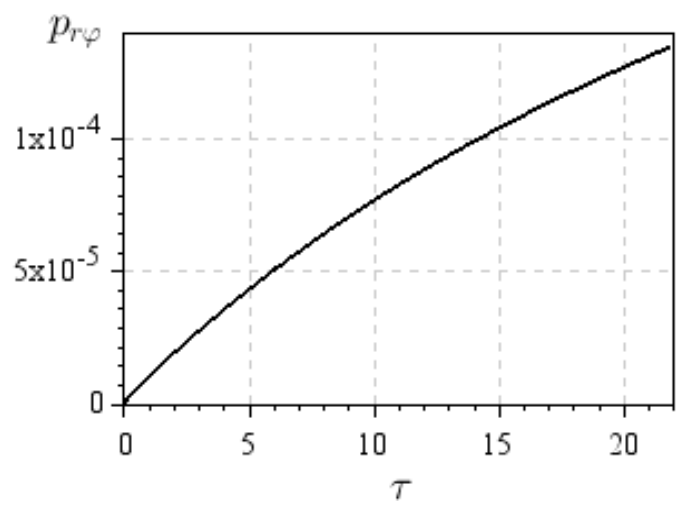

Рис. 2. Деформации ползучести при $r=r_{0}$ :

a-диагональные компоненты $p_{r r}$ и $p_{\varphi \varphi} ;$ б-недиагональная компонента $p_{r \varphi}$

Релаксация компонент $\sigma_{r \varphi}$ и наибольшей из диагональных по абсолютной величине $\sigma_{\varphi \varphi}$ тензора напряжений, проявляющаяся в снижении уровня напряжений после остановки вращающейся поверхности, показана на рис. 3.

a)

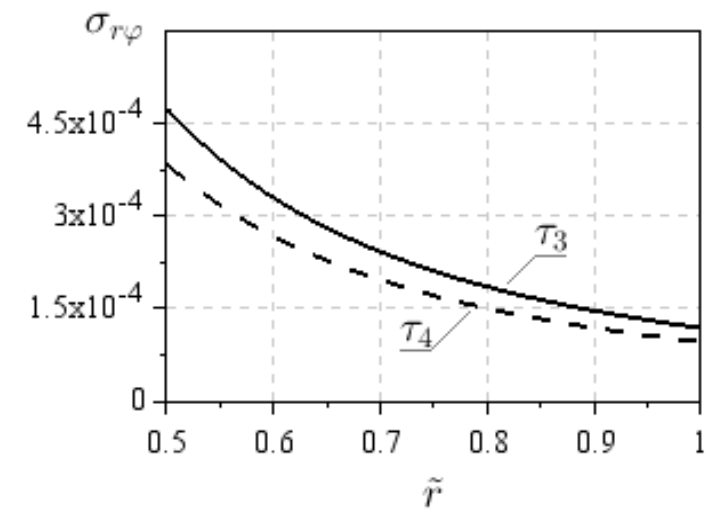

б)

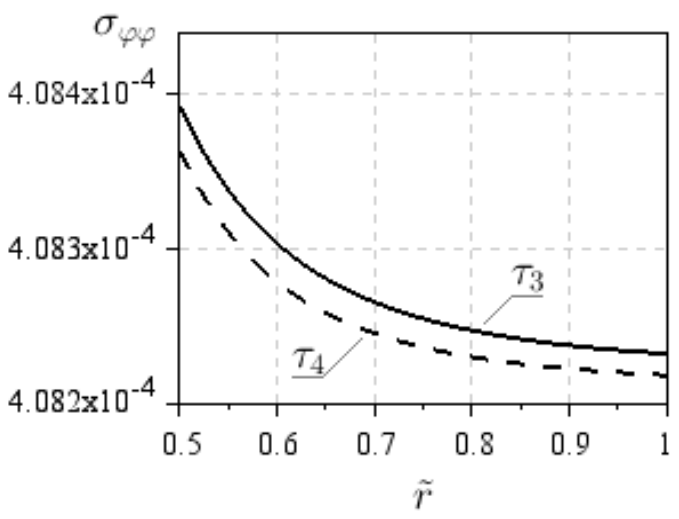

Рис. 3. Релаксация напряжений: $\mathrm{a}-$ компоненты $\sigma_{r \varphi} ; \sigma-$ компоненты $\sigma_{\varphi \varphi}$ 
Бегун А. С., Ковтанюк Л. В., Лемза А. О.

МОДЕЛИРОВАНИЕ ПРОЦЕССА ПРОИЗВОДСТВА БОЛЬШИХ ВИСКОЗИМЕТРИЧЕСКИХ ДЕФОРМАЦИЙ ВЯЗКОУПРУГОГО МАТЕРИАЛА

ПРИ ЕГО ОДНОСТОРОННЕМ ПРОСКАЛЬЗЫВАНИИ

\section{Вращение внутреннего цилиндра}

Рассмотрим теперь вариант задачи, при котором деформирование материала происходит при вращении внутреннего цилиндра радиуса $r=r_{0}$ и неподвижности внешней поверхности радиуса $r=R$. Кинематика среды в таком случае определяется, как и ранее, зависимостями (6). Скорость вращения внутреннего цилиндра зададим аналогично предыдущему варианту:

$$
w_{r_{0}}(t)=\left\{\begin{array}{lc}
\alpha^{2} t, & 0 \leq t \leq t_{1}, \\
\alpha^{2} t_{1}, & t_{1} \leq t \leq t_{2}, \\
\alpha^{2} t_{1}-\beta^{2}\left(t-t_{2}\right), & t_{2} \leq t \leq t_{3}, \\
0, & t_{3} \leq t .
\end{array}\right.
$$

Граничные условия в рассматриваемом случае принимают следующий вид:

$$
\left.\bar{u}\right|_{r=R}=\left.\overline{\mathrm{v}}\right|_{r=R}=0,\left.\quad \theta\right|_{r=r_{0}}=\theta_{r_{0}}(t)=\int_{0}^{t} w_{r_{0}}(\xi) d \xi,\left.\quad \sigma_{r r}\right|_{r=r_{0}}=\sigma_{0} .
$$

Соотношения (9)-(12), (16) и (18) при смене вращающейся поверхности не изменяются и действительны в рамках новой задачи. Однако некоторые входящие в них компоненты, а также дифференциальные уравнения для функций $c(t)$ и $c_{1}(t)$ будут отличаться от построенных выше.

Так, при интегрировании второго уравнения равновесия (12) получим

$$
\sigma_{r \varphi}=-\frac{c(t)}{r^{2}}, \quad e_{r \varphi}=-\frac{c(t)}{2 \mu r^{2}} .
$$

Отсюда для компоненты $\varepsilon_{r \varphi}^{v}$ тензора скоростей деформаций ползучести справедливо следующее соотношение:

$$
\varepsilon_{r \varphi}^{v}=\frac{\partial p_{r \varphi}}{\partial t}=-B n 2^{n-1} \frac{c^{n-1}(t)}{r^{2 n-2}}
$$
поворота

Аналогично зависимостям (15) и (17) в рассматриваемом случае получим уравнения для угла

$$
\theta(r, t)=\frac{c(t)}{2 \mu}\left(\frac{1}{r^{2}}-\frac{1}{R^{2}}\right)+\frac{B n 2^{n-1}}{n-1} c_{1}(t)\left(\frac{1}{r^{2 n-2}}-\frac{1}{R^{2 n-2}}\right)
$$

и функций $c(t)$ и $c_{1}(t)$ :

$$
\begin{aligned}
& \dot{c}(t)=\frac{2 \mu w_{r_{0}}(t)}{r_{0}^{-2}-R^{-2}}-\frac{\mu B n 2^{n}}{n-1} c^{n-1}(t) \cdot \frac{r_{0}^{2-2 n}-R^{2-2 n}}{r_{0}^{-2}-R^{-2}}, \\
& \dot{c}_{1}^{\frac{1}{n-1}}(t)=\frac{2 \mu \theta_{r_{0}}(t)}{r_{0}^{-2}-R^{-2}}-\frac{\mu B n 2^{n}}{n-1} c_{1}(t) \cdot \frac{r_{0}^{2-2 n}-R^{2-2 n}}{r_{0}^{-2}-R^{-2}} .
\end{aligned}
$$

Пусть в некоторый момент времени $t_{s}$ в окрестности $r=r_{0}$ неравенство (9) перестанет быть справедливым. Тогда новое граничное условие

$$
\left|\sigma_{r \varphi}\left(r_{0}, t\right)\right|=\gamma\left|\sigma_{r r}\left(r_{0}, t\right)\right|+\xi\langle w\rangle, \quad\langle w\rangle=w_{r_{0}}(t)-w\left(r_{0}, t\right),
$$


поставленное при $r=r_{0}$, приведёт к новому дифференциальному уравнению для функции $c(t)$ при $t_{s} \leq t \leq t_{a}$. Для получения такого уравнения сначала сформулируем определение угла поворота на основе (6) и (25), а затем и для угловой скорости точки среды:

$$
\begin{aligned}
& \theta(r, t)=\frac{c(t)}{2 \mu}\left(\frac{1}{r^{2}}-\frac{1}{R^{2}}\right)+\frac{B n 2^{n-1}}{n-1} c_{1}(t)\left(\frac{1}{r^{2 n-2}}-\frac{1}{R^{2 n-2}}\right), \\
& w(r, t)=\frac{\dot{c}(t)}{2 \mu}\left(\frac{1}{r^{2}}-\frac{1}{R^{2}}\right)+\frac{B n 2^{n-1}}{n-1} c^{n-1}(t)\left(\frac{1}{r^{2 n-2}}-\frac{1}{R^{2 n-2}}\right) .
\end{aligned}
$$

Тогда из (30) следует, что

$$
\dot{c}(t)=\frac{2 \mu}{r_{0}^{-2}-R^{-2}} \cdot\left[\frac{\gamma\left|\sigma_{0}\right|}{\xi}-\frac{c(t)}{\xi r_{0}^{2}}+w_{r_{0}}(t)\right]-\frac{\mu B n 2^{n}}{n-1} c^{n-1}(t) \frac{r_{0}^{2-2 n}-R^{2-2 n}}{r_{0}^{-2}-R^{-2}} .
$$

Начальным условием для (32) служит величина $c\left(t_{s}\right)$, найденная при решении (29). При $t \geq t_{a}$ функции $c(t)$ и $c_{1}(t)$ определяются решением уравнений (29) при начальном условии $c\left(t_{a}\right)$ и $c_{1}\left(t_{a}\right)$, вычисленных согласно (16) и (32).

Результаты расчёта задачи о вращении внутренней цилиндрической поверхности, проведённого, как и ранее, в безразмерных переменных при значениях параметров (23), представлены ниже.

На рис. 4 показано изменение угла поворота точек среды при $\tau=\tau_{1}, \tau_{2}, \tau_{3}, \tau_{4}$.

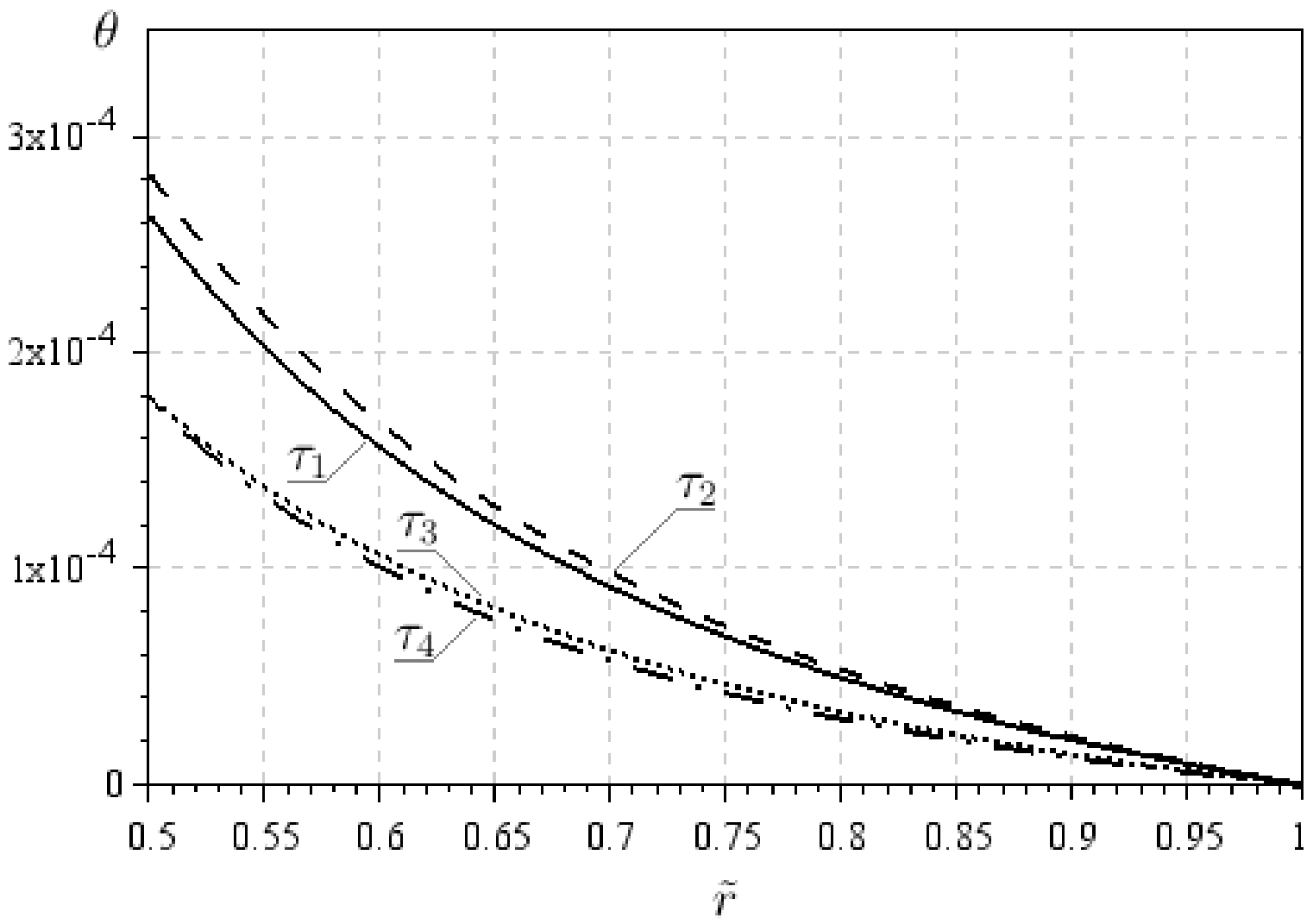

Рис. 4. Угол поворота $\theta(r, t)$ 
Бегун А. С., Ковтанюк Л. В., Лемза А. О.

МОДЕЛИРОВАНИЕ ПРОЦЕССА ПРОИЗВОДСТВА БОЛЬШИХ ВИСКОЗИМЕТРИЧЕСКИХ ДЕФОРМАЦИЙ ВЯЗКОУПРУГОГО МАТЕРИАЛА

ПРИ ЕГО ОДНОСТОРОННЕМ ПРОСКАЛЬЗЫВАНИИ

На рис. 5 проиллюстрировано изменение необратимых деформаций в ходе рассмотренного процесса в окрестности внутреннего цилиндра.

a)

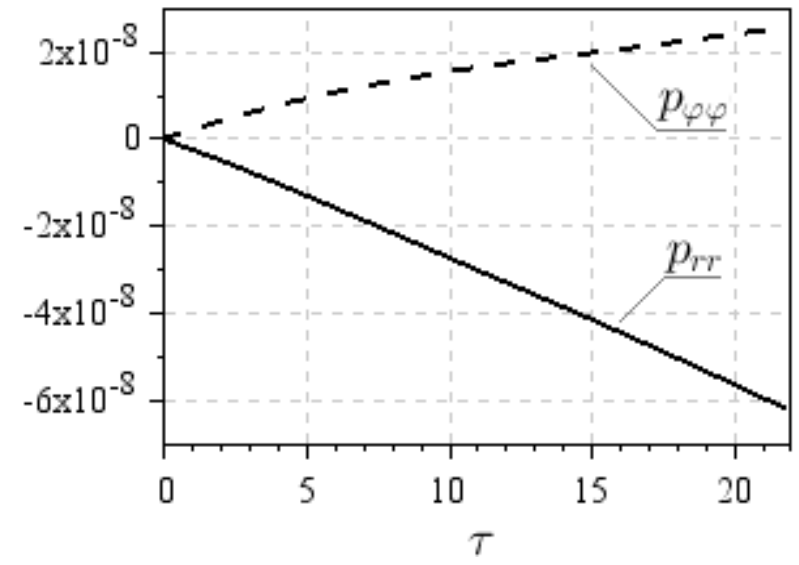

б)

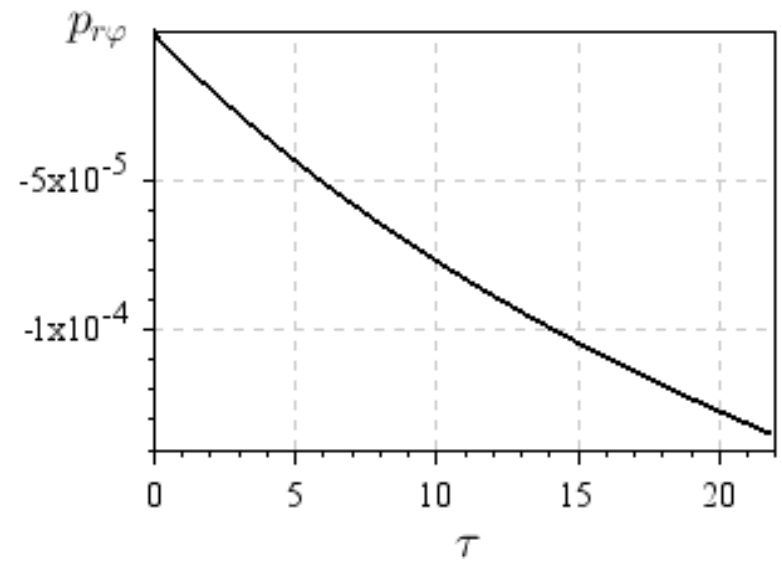

Рис. 5. Деформации ползучести при $r=r_{0}$ :

a - диагональные компоненты $p_{r r}$ и $p_{\varphi \varphi} ;$ б-недиагональная компонента $p_{r \varphi}$

Снижение уровня напряжений в течение длительного периода времени отражено на рис. 6.

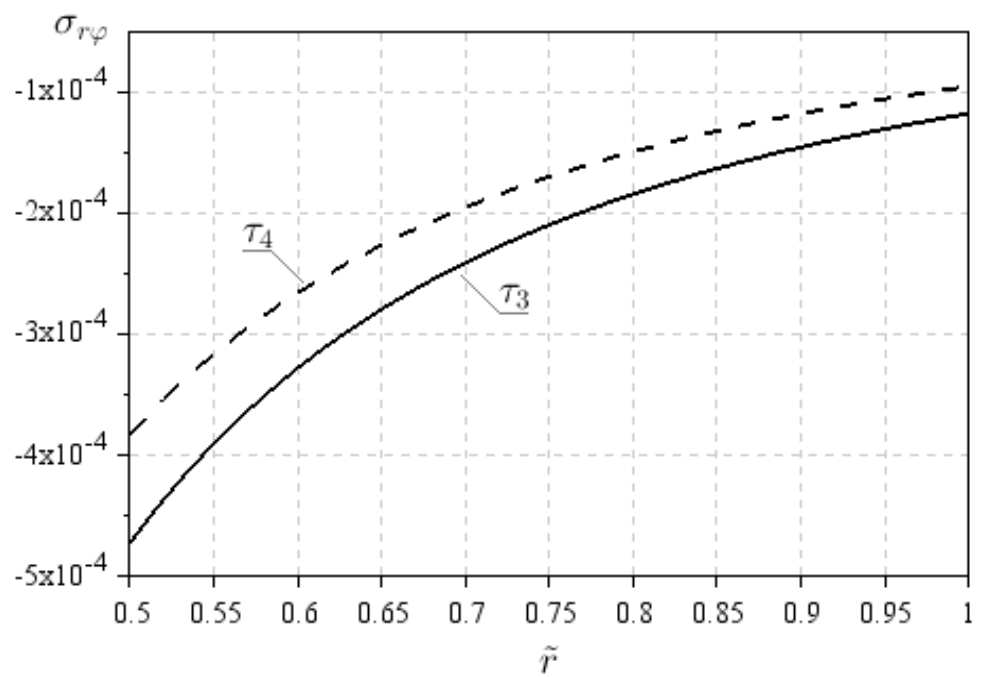

Рис. 6. Релаксация напряжений

\section{Заключение}

В рамках теории больших деформаций получено решение задачи о деформировании материала с нелинейными упругими и вязкими свойствами, находящегося в зазоре между двумя жёсткими коаксиальными цилиндрическими поверхностями при ротационном движении одной из жёстких поверхностей, в то время как вторая остаётся неподвижной. Изучено равноускоренное движение вращающегося цилиндра, последующие его движения с постоянной скоростью и дальнейшее равнозамедленное движение до остановки. Решения получены при условиях, когда в окрестности внутреннего жёсткого цилиндра возможно проскальзывание материала. Для случаев поворота внешней, а затем внутренней поверхностей рассчитаны напряжения, обратимые и необратимые деформации, перемещения; исследована релаксация напряжений после полной остановки цилиндра. 


\section{ЛИТЕРАТУРА}

1. Алексеев, Е. Р. Scilab: решение инженерных и математических задач / Е. Р. Алексеев, Е. А. Рудченко, O. В. Чеснокова. - М.: ALT Linux; БИНОМ. Лаборатория знаний, 2008. - 269 с.

2. Бегун, А. С. Течение упруговязкопластического материала между вращающимися цилиндрическими поверхностями в условиях нежёсткого сцепления / А. С. Бегун, А. А. Буренин, Л. В. Ковтанюк // Прикладная механика и техническая физика. - 2015. - Т. 56, № 2. - С. 146-158.

3. Бегун, А. С. Ползучесть и релаксация напряжений в материале цилиндрического слоя при его ротационном движении / А. С. Бегун, Л. В. Ковтанюк, А. О. Лемза // Вестник Чувашского государственного педагогического университета имени И. Я. Яковлева. Механика предельного состояния. - 2016. - № 4(30). C. 3-11.

4. Буренин, А. А. Об одной простой модели для упругопластической среды при конечных деформациях А. А. Буренин, Г. И. Быковцев, Л. В. Ковтанюк // Доклады Академии наук. - 1996. - Т. 347. - № 2. C. 199-201.

5. Буренин, А. А. Большие необратимые деформации и упругое последействие / А. А. Буренин, Л. В. Ковтанюк. - Владивосток: Дальнаука, 2013. - 312 с.

6. Лурье, А. И. Нелинейная теория упругости / А. И. Лурье. - М.: Наука, 1980. - 512 с.

7. Олейников, А. И. Интегрированное проектирование процессов изготовления монолитных панелей А. И. Олейников, А. И. Пекарш. - М.: Эком, 2009. - 112 с.

8. Norton, Frederick H. The creep of steel at high temperatures / H. Norton, Frederick. - Y.: McGrow Hill Book Company, 1929. - 110 p. 